\title{
Extracción de ADN de Trypanosoma cruzi mediante tratamiento con bromuro de hexadecil-trimetil-amonio
}

\author{
Marcela Escalante ${ }^{\text {, }}$ Marleny Montilla ${ }^{2}$, R. Santiago Nicholls ${ }^{2}$, \\ Patricia Del Portillo ${ }^{3}$, Concepción J. Puerta ${ }^{1}$
}

\begin{abstract}
Resumen
En el presente trabajo se describe un método rápido, sencillo y eficaz para la obtención de ADN genómico de Trypanosoma cruzi, libre de impurezas y fácil de manipular. Dicho procedimiento se basa en la lisis del parásito con SDS y remoción de proteínas mediante la digestión con proteinasa $\mathrm{K}$, seguida de la precipitación selectiva de carbohidratos y proteínas residuales con bromuro de hexadecil-trimetil-amonio (CTAB). Finalmente, el ADN se extrae con cloroformo: alcohol isoamílico y se recupera de la fase acuosa mediante precipitación con isopropanol.
\end{abstract}

\section{Summary}

The present work describes a fast, simple and efficient method of isolating pure and easily handling of genomic DNA from Trypanosoma cruzi. This protocol is based on parasite lysis with SDS and the removal of proteins by digestion with proteinase $\mathrm{K}$, followed by polysaccharides and remaining proteins' selective precipitation with CTAB. Finally, the DNA is extracted with chloroform-isoamyl alcohol and is recovered from the aqueous supernatant by isopropanol precipitation.

Trypanosoma cruzi, el agente etiológico de la enfermedad de Chagas, es un protozoo parásito que pertenece a la familia Trypanosomatidae, cuyos miembros se caracterizan no sólo por la presencia de estructuras únicas, como el cinetoplasto y el glicosoma, sino por la ocurrencia de mecanismos genéticos tales como la transcripción policistrónica, la transcripción discontinua y la edición del ARN mitocondrial (1-3).

Tradicionalmente, el interés en el estudio de los tripanosomátidos era de carácter médico y veterinario debido a las enfermedades ocasionadas por los mismos, como la enfermedad de Chagas (4), la leishmaniasis (5), la enfermedad del sueño (6) y la tripanosomiasis bovina (7). Actual- mente, dadas las características bioquímicas y genéticas peculiares de los miembros de esta familia, estos parásitos constituyen uno de los modelos más llamativos de investigación básica en el campo de la biología molecular.

Dentro del anterior contexto, surge la necesidad de encontrar métodos alternativos para la extracción de ADN de estos parásitos, que permitan agilizar procedimientos tales como el tamizaje de mutantes o transfectantes (8). Es así como, en el presente trabajo, se describe un procedimiento rápido, sencillo y eficaz, que permite la obtención de ADN libre de impurezas y fácil de manejar.

1 Departamento de Microbiología, Facultad de Ciencias, Pontificia Universidad Javeriana, Santa Fe de Bogotá.

${ }^{2}$ Laboratorio de Parasitología, Instituto Nacional de Salud, Santa Fe de Bogotá.

${ }^{3}$ Corporación Corpogen, Santa Fe de Bogotá. 


\section{Materiales y métodos}

Parásitos: se emplearon seis cepas colombianas de Trypanosoma cruzi (cuadro 1) en este estudio. La obtención de dichos parásitos en masa (200-300 mL) se realizó en medio líquido REI modificado (infusión de cerebro-corazón $10 \mathrm{~g} / \mathrm{L}$, $\mathrm{NaCl} 8 \mathrm{~g} / \mathrm{L}, \mathrm{KCl} 4 \mathrm{~g} / \mathrm{L}, \mathrm{MgSO} 4.7 \mathrm{H}_{2} \mathrm{O}$ 0,2 g/L, $\mathrm{Na}_{2} \mathrm{HPO}_{4} 0,06 \mathrm{~g} / \mathrm{L}, \mathrm{CaCl}_{2} 0,07 \mathrm{~g} / \mathrm{L}$, glucosa $2 \mathrm{~g} /$ $\mathrm{L}, \mathrm{NaHCO}_{3} 1 \mathrm{~g} / \mathrm{L}$ ) suplementado con $2 \%$ de suero fetal bovino (SFB) y $100 \mathrm{mg} / \mathrm{mL}$ de gentamicina, a $24^{\circ} \mathrm{C}(9)$.

Extracción del ADN: una vez obtenida la masa de parásitos en fase logarítmica de crecimiento (200-300 mL), se recolectó mediante centrifugación a 3.000 rpm durante $20 \mathrm{~min}$, y se lavó con solución tampón TE (tris-HCl 10 mM, EDTA $1 \mathrm{mM}, \mathrm{pH} 7,4)$. Tras la última centrifugación, los parásitos fueron resuspendidos en solución tampón de lisis a una concentración final de SDS al $1 \%$ y proteinasa K (Sigma) a $25 \mu \mathrm{g} / \mathrm{mL}$, e incubados durante $1 \mathrm{~h}$ a $65^{\circ} \mathrm{C}$. Posteriormente, la suspensión se ajustó a $0,7 \mathrm{M}$ de $\mathrm{NaCl}$ y se adicionó una mezcla de bromuro de hexadecil-trimetilamonio (CTAB) (Sigma) al $10 \%$ en $0,7 \mathrm{M} \mathrm{de} \mathrm{NaCl}$, durante $20 \mathrm{~min}$. a $65^{\circ} \mathrm{C}$. Transcurrido este perío- do, el ADN fue extraido (V:V) con cloroformoisoamílico $24: 1$ y precipitado con 6 volúmenes de isopropanol. Finalmente, la madeja de ADN obtenida se lavó con etanol al $70 \%$ y se resuspendió en TE.

EI ADN extraido se analizó mediante electroforesis horizontal en geles de agarosa al $1 \%$, utilizando TAE como solución tampón (tris- $\mathrm{HCl} 0,04 \mathrm{M} \mathrm{pH}$ 7,2, ácido acético glacial 29,6 mM y EDTA 2 mM $\mathrm{pH} 8$ ), con posterior tinción con bromuro de etidio (Sigma), a una concentración final de $0,5 \mu \mathrm{g} / \mathrm{mL}$ de agua destilada (10).

Finalmente, la concentración del ADN obtenido se midió por espectrofotometría a UV a $260 \mathrm{~nm}$, teniendo en cuenta que 1 unidad de densidad óptica (DO) de $260 \mathrm{~nm}$ equivale a $50 \mu \mathrm{g} / \mathrm{mL}$ de $A D N$ de doble cadena (10).

Digestión del ADN de los parásitos: se digirieron $2 \mu \mathrm{g}$ de ADN con diferentes endonucleasas de restricción, utilizando una relación de 10:1 de enzima/ $\mu \mathrm{g}$ de ADBN. Las hidrólisis se realizaron en un volumen de $30 \mu \mathrm{L}$, durante $16 \mathrm{~h}$ a $37^{\circ} \mathrm{C}$.

Estudios de Southern blot: una vez digerido el ADN, los fragmentos resultantes fueron someti-

Cuadro 1. Características de las cepas de Trypanosoma cruzi en estudio.

\begin{tabular}{|c|c|c|c|c|}
\hline Cepa & Código & Procedencia & Aislamiento & Origen \\
\hline 1 & $\mathrm{MHOM} / \mathrm{CO} / 86$ & $\begin{array}{c}\text { Chiriguaná } \\
\text { Cesar }\end{array}$ & Hemocultivo & Humano \\
\hline 2 & $\mathrm{MHOM} / \mathrm{CO} / 87$ & $\begin{array}{l}\text { Paratebueno, } \\
\text { Cundinamarca }\end{array}$ & Hemocultivo & Humano \\
\hline Munanta & $\begin{array}{c}\text { IRHO/CO/85 } \\
\text { Munanta }\end{array}$ & $\begin{array}{c}\text { Munanta, } \\
\text { Guateque, } \\
\text { Boyacá }\end{array}$ & $\begin{array}{l}\text { Materia fecal } \\
\text { de pito }\end{array}$ & $\begin{array}{l}\text { R. prolixus } \\
\text { Domiciliario }\end{array}$ \\
\hline Shubacbarina & $\begin{array}{l}\text { IRHO/CO/95 } \\
\text { Shubacbarina }\end{array}$ & $\begin{array}{l}\text { Shubacbarina } \\
\text { Catatumbo } \\
\text { Norte de Santander }\end{array}$ & $\begin{array}{l}\text { Materia fecal } \\
\text { de pito }\end{array}$ & $\begin{array}{l}\text { R. prolixus } \\
\text { Silvestre }\end{array}$ \\
\hline Ikiakarora & $\begin{array}{l}\text { IRHO/CO/95 } \\
\text { Ikiakarora }\end{array}$ & $\begin{array}{c}\text { Ikiakarora } \\
\text { Catatumbo } \\
\text { Norte de Santander }\end{array}$ & $\begin{array}{l}\text { Materia fecal } \\
\text { de pito }\end{array}$ & $\begin{array}{l}\text { R. prolixus } \\
\text { Silvestre }\end{array}$ \\
\hline $\begin{array}{l}\text { D. marsupialis } \\
\text { No } 3\end{array}$ & $\begin{array}{c}\text { MDID/CO/87 } \\
\text { No.3 }\end{array}$ & $\begin{array}{c}\text { Astilleros } \\
\text { El Zulia, } \\
\text { Norte de Santander }\end{array}$ & Hemocultivo & $\begin{array}{l}\text { D. marsupialis } \\
\text { Silvestre }\end{array}$ \\
\hline $\begin{array}{l}\text { D. marsupialis } \\
\text { R-56 }\end{array}$ & $\begin{array}{c}\mathrm{MDID} / \mathrm{CO} / 88 \\
\mathrm{R}-56\end{array}$ & $\begin{array}{c}\text { El Callejón } \\
\text { Ricaurte, } \\
\text { Cundinamarca }\end{array}$ & Hemocultivo & $\begin{array}{l}\text { D. marsupialis } \\
\text { Silvestre }\end{array}$ \\
\hline
\end{tabular}


dos a electroforesis horizontal en geles de agarosa al 0,8\% y transferidos a membranas de nylon neutras (Magnagraph MSI, Fisher), siguiendo el protocolo de transferencia salina, descrito por Southern en 1975. Posteriormente, los filtros fueron sometidos a una reacción de prehibridación durante $2 \mathrm{~h}$ a $42^{\circ} \mathrm{C}$ en $50 \%$ de formamida, $0,12 \mathrm{M}$ de $\mathrm{Na}_{2} \mathrm{HPO}_{4} \mathrm{pH} 7,2,0,25 \mathrm{M} \mathrm{NaCl}, 7 \%$ SDS, 1 mM EDTA y $200 \mu \mathrm{g}$ de ADN de esperma de salmón denaturado, por $\mathrm{mL}$ de solución. Seguidamente, los filtros fueron hibridados con el gen que codifica para la histona H2A de T. cruzi (12), marcado con biotina y previamente denaturado, en una solución nueva de la mezcla anterior, a $42^{\circ} \mathrm{C}$ durante $24 \mathrm{~h}$.

Transcurrida la hibridación, los filtros se enjuagaron brevemente en una solución de SSC 2X-SDS al $0,5 \%$ (p/v) a temperatura ambiente y se lavaron durante $15 \mathrm{~min}$ a temperatura ambiente y agitación constante, con cada una de las siguientes soluciones: SSC $1 \mathrm{X}$ - SDS al $0,5 \%(\mathrm{p} / \mathrm{v})$, SSC $0,5 \mathrm{X}-\mathrm{SDS}$ al $0,5 \%(\mathrm{p} / \mathrm{v})$ y SSC $0,1 \mathrm{X}-\mathrm{SDS}$ al $0,5 \%(\mathrm{p} / \mathrm{v})$.

Finalmente, una vez se secaron los filtros, se procedió a la reacción de detección con el conjuga- do fosfatasa alcalina-estreptavidina, según lo recomendado en el manual del Photogen Detection System (Gibco-BRL), seguido del revelado de las señales con el sustrato NBT/BCIP (Gibco-BRL).

\section{Resultados}

\section{Obtención del ADN de los parásitos}

Los ADN genómicos de las distintas cepas de $T$. cruzien estudio fueron extraidos según lo indicado en materiales y métodos. En la figura 1 se aprecia el grado de pureza de los distintos ADN y la ausencia de ARN. Este resultado está de acuerdo con la relación obtenida de absorbancia $\mathrm{A}_{260 /}$ ${ }_{280}$, (10), la cual se ubica dentro de los rangos permitidos $(1,8$ a 2,0$)$ en todas las muestras obtenidas. De otra parte, el rendimiento obtenido con este método es bastante alto ya que se obtiene alrededor de $1 \mu \mathrm{g}$ de $A D N$ por cada $\mathrm{mL}$ de cultivo crecido en fase exponencial ( $10^{6}$ parásitos $/ \mathrm{mL}$ ).

\section{Digestión del ADN con enzimas de restricción}

Los ADN extraidos fueron fácilmente digeridos en su totalidad con diversas endonucleasas que presentan diferentes longitudes en sus dianas de restricción. Bam HI, EcoR I, Hind III, Kpn I y Sph I,
Figura 1. Electroforesis horizontal en geles de agarosa al $1 \%$ del ADN genómico de los parásitos, teñido con bromuro de etidio: 1) marcador de peso molecular, $1 \mathrm{~Kb}$ ladder (Gibco-BRL); 2) ADN de la cepa 2; 3) ADN de la cepa 3 ; 4) ADN de la cepa $D$. marsupialis No. 3 ; 5) ADN de la cepa $D$. marsupialis R-56; 6) ADN de la cepa Shubacbarina; y 7) ADN de la cepa Ikiakarora.

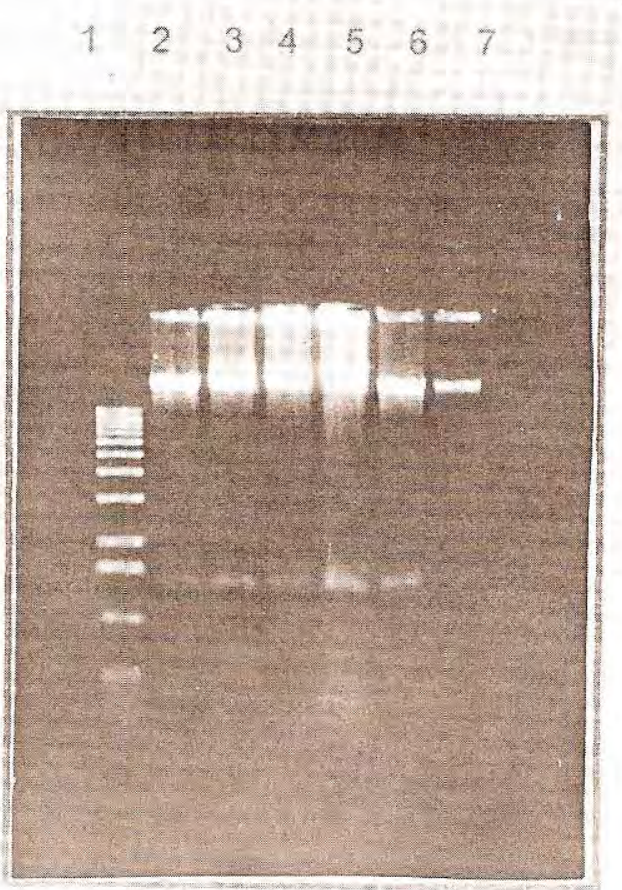


con cinco nucleótidos en su diana, BstE II, con siete nucleótidos y Not I con ocho nucleótidos. En la figura 2, se muestran los resultados obtenidos tras la digestón con la endonucleasa BstE II.

\section{Southern blot}

En la figura 3, se puede apreciar el patrón característico de restricción de los genes codificantes para la histona $\mathrm{H} 2 \mathrm{~A}$ de $T$. cruzi, con la enzima Sph I, consistiendo en las señales de hibridación correspondientes a las unidades de 0,76 y $1,2 \mathrm{~kb}$ (12).

\section{Discusión}

La mayoría de los protocolos empleados para la obtención de ADN genómico de tripanosomátidos consiste en la lisis del parásito con detergentes en la presencia de proteasas (13-15) o con agentes denaturantes como el cloruro de guanidina (16), seguida de una serie de extracciones con fenol, fenol:cloroformo:alcohol isoamílico y cloroformo: alcohol isoamílico y recuperación del ADN mediante precipitación con etanol.

En este trabajo, se realizaron algunas modificaciones tales como el tratamiento de los parásitos lisados con CTAB. Este detergente tiene la propiedad de formar complejos con polisacáridos y proteínas (17), de manera que su unión a las proteínas residuales y grupos carbohidratos presentes en los parásitos lisados permite la obtención de un ADN libre de contaminantes que, en un momento dado, pueden afectar la calidad del mismo, al interferir con la actividad de las endonucleasas de restricción, cinasas, ligasas y otras enzimas de uso frecuente en biología molecular.

Adicionalmente, el procedimiento descrito en este trabajo obvia pasos como las extracciones con fenol y fenol:cloroformo:alcohol isoamílico, de for-

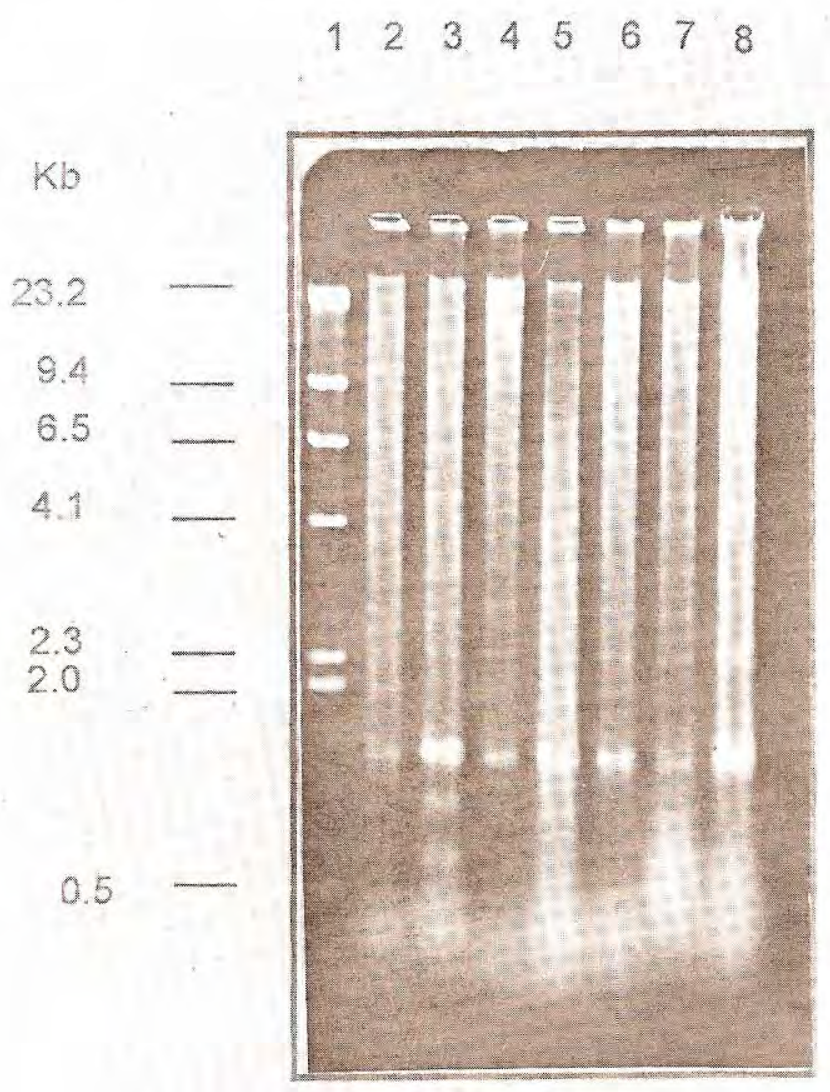

Figura 2. Electroforesis horizontal en geles de agarosa al $0,8 \%$ del ADN genómico de los parásitos digeridos con BstE II: 1) patrón de peso molecular, fago ëgt11 digerido con Hind III (Gibco-BRL); 2) ADN de la cepa 2; 3) ADN de la cepa 3 ; 4) ADN de la cepa $D$. marsupialis No. 3; 5) ADN de la cepa D. marsupialis R-56; 6) ADN de la cepa Shubacbarina; 7) ADN de la cepa Ikiakarora; y 8) ADN de la cepa Munanta. 


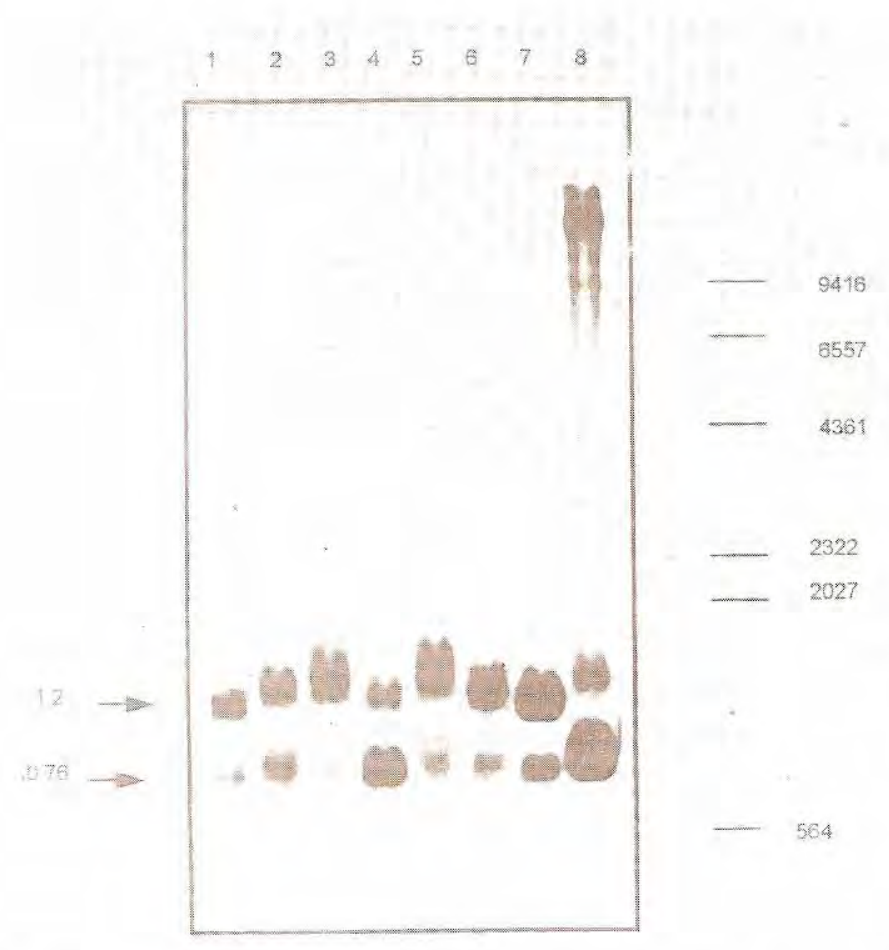

Figura 3. Estudio de RFLP (restriction fragment length polymorphisms) después de la digestión con Sphl. $2 \mu \mathrm{g}$ de ADN de epimastigotes de $T$. cruzi de las cepas: 1) Shubacbarina; 2) Munanta; 3) cepa 3; 4) Ikiakarora; 5) D. marsupialis R-56; 6) D. marsupialis No. 3; 7) cepa 2; y 8) Y, fueron digeridos con la enzima Sphl e hibridados con la unidad $1,2 \mathrm{~Kb}$ codificante para la histona $\mathrm{H} 2 \mathrm{~A}$ de dicho parásito, marcada con biotina. A la derecha, se indican los tamaños, en $\mathrm{Kb}$, de los fragmentos Hindlll del fago Lambda gt11. ma que no sólo se reduce el tiempo, sino que se evita el uso de reactivos tóxicos y corrosivos como el fenol, cuya manipulación requiere del uso de campanas de extracción. Así mismo, en el protocolo propuesto, a diferencia de los referidos anteriormente, no se requiere del tratamiento con ARNasas, de tal forma que se acorta aún más el tiempo del procedimiento, así como también los gastos del mismo.

A diferencia de la mayoría de los procedimientos para extraer ADN genómico de tripa-nosomátidos (13-16) que precipitan el mismo con etanol, en el método que se describe, dada la eliminación previa de carbohidratos y proteínas residuales, se utiliza isopropanol, permitiendo así la obtención de una cantidad considerable de $\operatorname{ADN}\left(1 \mu \mathrm{g} / 10^{6}\right.$ parásitos).

El tratamiento con CTAB ha sido ampliamente utilizado en el aislamiento de ADN cromosómico de bacterias gramnegativas como Rhizobium (18) y Chlamydia pneumoniae (19) y de Mycobacterias (20), las cuales normalmente producen grandes cantidades de polisacáridos, así como también en la obtención de ADN de alto peso molecular en plantas (21). T.cruzi es un proto- zoario que posee una gran cantidad de glicoconjugados en su membrana, tales como oligosacáridos presentes en complejos como el lipidopéptido glicano y glicoproteínas, algunas de las cuales son exportadas al medio (22-23), de manera que la extracción de ADN con CTAB, remueve estos polisacáridos, logrando un ADN libre de contaminantes y fácil de manipular, en un tiempo relativamente corto, lo cual permite un análisis ágil de mutantes o transfectantes, así como también la utilización del ADN obtenido en ensayos de Southern blot y PCR, entre otros.

\section{Agradecimientos}

A la doctora Genoveva Keyeux, del Instituto de Genética de la Pontificia Universidad Javeriana, por el préstamo de las cámaras fotográficas y transiluminador UV de su laboratorio, así como al doctor Jaime Bernal del Departamento de Química de la Pontificia Universidad Javeriana, por su colaboración en el uso de equipos de laboratorio.

Este trabajo se realizó con el apoyo financiero de la Fundación para la Promoción de la Investigación y la Tecnología del Banco de la República, Santa Fe de Bogotá, Colombia. 


\section{Referencias}

1. Thomas MC, Fernández A, Moro A, García JA, González A. Biología molecular de los tripanosomátidos. En: López MC, Rivas L, editores. Parasitología molecular: nuevas tendencias. Madrid: CSIC; 1993:135-43.

2. Vanhamme L, Pays E. Control of gene expression in Trypanosomes. Microbiol Rev 1995;6:223-40.

3. Boothroyd JC. Molecular biology of Trypanosomes. In: Wiley DJ, editor. Modern parasite: biological, cellular, immunological and molecular aspects. New York: W. H. Freeman; 1990:333-47.

4. Tanowitz HB, Kirchhoff LV, Simon D, Morris SA, Weiss LM, Witner M. Chagas' disease. Clin Microbiol Rev 1992;5:400-19.

5. Chang KP, Chaudhuri G. Molecular determinants of Leishmania virulence. Ann Rev Microbiol 1990; 44:499-529.

6. Cross GAM. Cellular and genetic aspects of antigenic variation in Trypanosomes. Ann Rev Immunol 1990;8:83-110.

7. Dirie MF, Otte MJ, Thatthi R, Gardiner PR. Comparative studies of Trypanosoma (Dutonella) vivax isolates from Colombia. Parasitology 1993; 106:21-9.

8. Parsons M. Current concepts in stage-regulated gene expression in Kinetoplastida. Parasitol Today 1990;6:244-7.

9. Duque S, Peláez D, Corredor A. Normas para el cultivo in vitro de parásitos de la familia Tripanosomatidae. Manual de procedimientos. Santa $\mathrm{Fe}$ de Bogotá: Instituto Nacional de Salud; 1993:41-5.

10. Maniatis T, Fritsh EF, Sambroock J. Gel electrophoresis of DNA. In: Molecular cloning. A laboratory manual. Second edition. Cold Spring Harbor: Cold Spring Harbor Laboratory; 1989:6.019.

11. Southern EM. Detection of specific sequences among DNA fragments separated by gel electrophoresis. J Mol Biol 1975;98:503.

12. Puerta CJ, Martín J, Alonso C, López MC. Isolation and characterization of the gene encoding histone H2A from Trypanosoma cruzi. Mol Biochem Parasitol 1994;64:1-10.

13. Requena JM, López MC, Jiménez A, De la Torre JC, Alonso A. A head tandem organization of hsp70 genes in Trypanosoma cruzi. Nucl Acids Res 1988;16:1393-1406.

14. Ismail SO, Skeiky YAW, Bhatia A, Omara-Opyene LA, Gedamu L. Molecular cloning, characterization, and expression in Escherichia coli of iron superoxide dismutase cDNA from Leishmania donovani chagasi. Infect Immun 1994; 62:657-64.

15. Steinkraus HB, Greer JM, Stephenson DC, Langer, PJ. Sequence heterogeneity and polymorphic gene arrangements of the Leishmania guyanensis gp63 genes. Mol Biochem Parasitol 1993;62:173-86.

16. Bock JH, Langer, PJ. Sequence and genomic organization of the hsp70 genes of Leishmania amazonensis. Mol Biochem Parasitol 1993;62:18798.

17. Wilson K. Preparation of genomic DNA from bacteria. In: Ausbel FM, Brent R, Kingston RE, Moore DD, Smith JA, Seidman JD, Struhl K, editors. Current protocols in molecular biology. New York: Greene Publishing and Wiley Interscience; 1988;2.4.2-4.5.

18. Meade HM, Long SR, Ruvkun CB, Brown SE, Ausubel FM. Physical and genetic characterization of symbiotic and auxotrophic mutants of Rhizobium meliloti induced by transposon Tn5 mutagenesis. J Bacteriol 1982;149:114-22.

19. Maass M, Dalhoff $\mathbf{K}$. Comparison of sample preparation methods for detection of Chlamydia pneumoniae in bronchoalveolar lavage fluid by PCR. J Clin Microbiol 1994;32:2626-9.

20. Parra CA, Londoño LP, Del Portillo P, Patarroyo ME. Isolation, characterization, and molecular cloning of a specific Mycobacterium tuberculosis antigen gene: identification of a species-specific sequence. Infect Immun 1991;59:3411-7.

21. Murray MG, Thompson WF. Rapid isolation of high-molecular-weight plant DNA. Nucl Acids Res 1980;8:4321-5.

22. Mendoza-Previato L, Gorin PAJ, Braga AF, Scharfstein J, Previato, JA. Chemical structure and antigenic aspects of complexes obtained from epimastigotes of Trypanosoma cruzi. Biochemestry 1983;22:4980-7.

23. Pereira MEA. Biology of Trypanosoma cruzi. In: Wiley DJ, editor. Modern parasite: biological, cellular, immunological and molecular aspects. New York: W. H. Freeman; 1990:65-261. 